\title{
Enhancement of production of eugenol and its glycosides in transgenic aspen plants via genetic engineering.
}

\section{$\operatorname{AUTHOR}(\mathrm{S})$ :}

Koeduka, Takao; Suzuki, Shiro; lijima, Yoko; Ohnishi, Toshiyuki; Suzuki, Hideyuki; Watanabe, Bunta; Shibata, Daisuke; Umezawa, Toshiaki; Pichersky, Eran; Hiratake, Jun

\section{CITATION:}

Koeduka, Takao ...[et al]. Enhancement of production of eugenol and its glycosides in transgenic aspen plants via genetic engineering.. Biochemical and biophysical research communications 2013, 436(1): 73-78

\section{ISSUE DATE:}

2013-06-21

URL:

http://hdl.handle.net/2433/175512

\section{RIGHT:}

(c) 2013 Elsevier Inc.; 許諾条件により本文は2013-11-01に公開.; This is not the published version. Please cite only the published version.; この 論文は出版社版でありません。引用の際には出版社版をご確認ご利用 ください。 
1 Title:

2 Enhancement of production of eugenol and its glycosides in transgenic aspen plants via

3 genetic engineering

4

5 Authors:

6 Takao Koeduka ${ }^{\mathrm{a}, 1, *}$, Shiro Suzuki ${ }^{\mathrm{b}, 1}$, Yoko Iijima ${ }^{\mathrm{c}}$, Toshiyuki Ohnishi ${ }^{\mathrm{d}}$, Hideyuki

$7 \quad$ Suzuki $^{\mathrm{e}}$, Bunta Watanabe ${ }^{\mathrm{a}}$, Daisuke Shibata ${ }^{\mathrm{e}}$, Toshiaki Umezawa ${ }^{\mathrm{b}}$, Eran Pichersky ${ }^{\mathrm{f}}$, Jun

8 Hiratake $^{\mathrm{a}}$

9

10 Affiliation:

11 Institute for Chemical Research, Kyoto University, Uji, Kyoto, 611-0011, Japan

$12{ }^{\mathrm{b}}$ Research Institute for Sustainable Humanosphere, Kyoto University, Uji, Kyoto

13 611-0011, Japan

$14{ }^{c}$ Faculty of Applied Bioscience, Kanagawa Institute of Technology, 1030 Shimo-ogino,

15 Atsugi, Kanagawa 243-0292, Japan

$16{ }^{\mathrm{d}}$ Faculty of Agriculture, Shizuoka University, 836 Ohya, Suruga-ku, Shizuoka 422-8529,

17 Japan

18 'Kazusa DNA Research Institute, 2-6-7 Kazusakamatari, Kisarazu, Chiba 292-0818,

19 Japan

$20{ }^{\mathrm{f}}$ Department of Molecular, Cellular and Developmental Biology, University of

21 Michigan, 830 North University Street, Ann Arbor, MI 48109-1048, USA

22

$23{ }^{1}$ These authors contributed equally to this work.

24

25 *To whom correspondence should be addressed:

26 Institute for Chemical Research, Kyoto University, Uji, Kyoto 611-0011, Japan

27 Tel: +81774383230 
$1 \quad$ Fax: +81 774383229

2 E-mail: takaori@scl.kyoto-u.ac.jp

3

4

5

6

7

8

9

10

11

12

13

14

15

16

17

18

19

20

21

22

23

24

25

26

27 


\section{Abstract}

2 Eugenol, a volatile phenylpropene found in many plant species, exhibits antibacterial

3 and acaricidal activities. This study attempted to modify the production of eugenol and

4 its glycosides by introducing petunia coniferyl alcohol acetyltransferase (PhCFAT) and

5 eugenol synthase (PhEGS) into hybrid aspen. Gas chromatography analyses revealed

6 that wild-type hybrid aspen produced small amount of eugenol in leaves. The

7 heterologous overexpression of PhCFAT alone resulted in up to 7-fold higher eugenol

8 levels and up to 22-fold eugenol glycoside levels in leaves of transgenic aspen plants.

9 The overexpression of $P h E G S$ alone resulted in a subtle increase in either eugenol or

10 eugenol glycosides, and the overexpression of both PhCFAT and PhEGS resulted in

11 significant increases in the levels of both eugenol and eugenol glycosides which were

12 nonetheless lower than the increases seen with overexpression of PhCFAT alone. On the

13 other hand, overexpression of PhCFAT in transgenic Arabidopsis and tobacco did not

14 cause any synthesis of eugenol. These results indicate that aspen leaves, but not

15 Arabidopsis and tobacco leaves, have a partially active pathway to eugenol that is

16 limited by the level of CFAT activity and thus the flux of this pathway can be increased

17 by the introduction of a single heterologous gene.

19 Keywords

20 transgenic aspen; phenylpropene; monolignol; eugenol glycoside; metabolic 21 engineering

\section{Introduction}

24 Eugenol belongs to the class of volatile phenylpropenes that are widely distributed 25 across the plant kingdom. Many phenylpropenes impart a characteristic aroma, and 26 some fresh fruits and processed products that contain them are widely consumed. For 27 example, in the important vegetable crop tomato, many phenylpropene volatiles, 
1 including eugenol, are often stored as glycosides, representing an aroma reserve, and

2 overall content of releasable phenylpropenes is an important factor determining the

3 commercial value of the tomato fruits [1,2]. Volatile phenylpropenes also exhibit

4 various physiological activities, antimicrobial and acaricidal activities [3,4], thus these

5 compounds function as direct defense against microorganisms and herbivorous pests.

6 Eugenol is biosynthesized via two enzymatic steps. Coniferyl alcohol

7 acetyltransferase (CFAT) catalyzes the first step in eugenol biosynthesis that branches

8 off from the lignin pathway, and eugenol synthase (EGS) is the second and last enzyme

9 responsible for synthesis of eugenol (Fig. 1). The two genes have been isolated and

10 functionally characterized in basil (Ocimum basilicum), petunia (Petunia hybrida and

11 Petunia axillaris), Clarkia breweri, and anise (Pimpinella anisum) [5-10].

12 To date, genetic modification of phenylpropene biosynthesis has been used to alter

13 the volatile composition only in strawberry fruits [11]. In transgenic strawberry, the

14 modification of anthocyanin biosynthesis by simultaneously expressing $E G S$ and

15 downregulating chalcone synthase (CHS) gene boosted the formation of eugenol,

16 isoeugenol, and their analogs chavicol and anol (the latter two are phenylpropenes

17 without a functional group at $3^{\prime}$ position of the aromatic ring, see Fig. 1), indicating that

18 diverting the flavonoid pathway to phenylpropene biosynthesis through metabolic

19 engineering [11] is also possible. Surprisingly, there is no report about the metabolic

20 engineering in vegetative parts, using model plants such as tobacco and Arabidopsis.

21 Hybrid aspen (Populus tremula $\times$ tremuloides), which is a relatively amenable tree

22 species for transformation, has been utilized to genetically engineer the lignin

23 biosynthetic pathway. Because the lignin pathway shares some of its precursors with the

24 phenylpropene pathway (Fig. 1) and enhancing the productivity of eugenol in planta

25 may contribute to improve plant protection, plants with high levels of lignin

26 biosynthesis are attractive targets for genetic manipulation of the phenylpropene

27 pathway. In this study, we attempted to engineer eugenol synthesis in tobacco, 
1 Arabidopsis, and hybrid aspen by introducing petunia $C F A T$ and/or petunia $E G S$ genes.

2

3 Materials and methods

$4 \quad$ Preparation and growth of transgenic plants

5 In preparation of transgenic hybrid aspen, tobacco and Arabidopsis, we first subcloned

6 the coding sequences of P. hybrida CFAT (PhCFAT; GenBank accession ABG75942)

7 and P. hybrida EGS (PhEGS; GenBank accession ABR24115) individually into the

8 pENTR/D-TOPO vector (Life Technologies, Carlsbad, CA). The resulting entry vector

9 was then recombinated with the destination vector pH35GS [12] or pMDC32 [13] using

10 LR clonase II (Life Technologies). Agrobacterium-mediated transformation and

11 regeneration of hybrid aspen (Populus tremula $\times$ tremuloides T89) were carried out as

12 previously described [12] using the equivalent mixture of cells [14] of A. tumefaciens

13 GV3101::pMP90 harboring pH35GS-PhCFAT and pH35GS-PhEGS (Supplementary

14 Fig. 1). The regenerated transformants were screened by RT-PCR with the specific 15 primers described as below. PCR-positive transgenic aspen were acclimatized in a 16 growth chamber at $25^{\circ} \mathrm{C}$ under a 16 -h light/8-h dark photoperiod for one month. The 17 plants were then transplanted into $1 / 10,000 \mathrm{a}$ Wagner pots containing a equivalent 18 mixture of garden soil "Hanasaki Monogatari" (Akimoto-tensanbutsu, Iga, Japan) and 19 vermiculite and were grown in a greenhouse at $25 \pm 2{ }^{\circ} \mathrm{C}$ from June to October. The 20 transgenic Arabidopsis and tobacco plants were produced by using A. tumefaciens strain 21 AGL1 harboring pMDC32-PhCFAT (Supplementary Fig. 1) and a floral dip or a leaf 22 disc protocol $[15,16]$. The obtained transformants were grown in the growth chamber at $2322^{\circ} \mathrm{C}$ for Arabidopsis and $25^{\circ} \mathrm{C}$ for tobacco under a 14 -h light/10-h dark photoperiod.

$25 \quad R T-P C R$ analysis

26 Total RNA was isolated from each leaf tissue with an RNeasy Plant Mini Kit (Qiagen, 27 Tokyo, Japan). The RNA was subjected to DNase treatment using the DNA-free kit 
1 (Life Technologies), and the first strand cDNAs were synthesized using Superscript III

2 reverse transcriptase (Life Technologies) with Oligo-dT primer. Semi-quantitative

3 RT-PCR experiments with KOD FX Neo polymerase (Toyobo, Tokyo, Japan) were

4 carried out for 32 cycles $\left(98^{\circ} \mathrm{C}\right.$ for $2 \mathrm{~min}$ and then cycling at $98^{\circ} \mathrm{C}$ for $15 \mathrm{sec}, 54^{\circ} \mathrm{C}$ for

$530 \mathrm{sec}$ and $68^{\circ} \mathrm{C}$ for $30 \mathrm{sec}$ ). PCR primers for PhCFAT amplification were forward

6 primer, 5'-TATTGACGATTCTAAAAGATGCAAACTT-3', and reverse primer,

7 5'-TAtTGACGATTCTAAAAGATGCAAACTT-3'. Primers (forward and reverse,

8 respectively) for PhEGS were 5'-CCAACTTTGGTTCAGCCAGGAGC-3' and

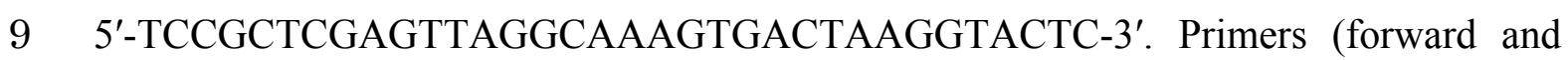

10 reverse, respectively) for Actin, which was used as reference for standardization of

11 cDNA amounts, were 5'-GCCAGTGGTCGTACAACTGGTATTG-3' and

12 5'-CCTTGATCTTCATGCTTGGAGC-3'.

13

14 Analysis of phenylpropene volatiles

15 Leaves (250 mg fresh weight) from each plant were ground using mortar and pestle in

16 liquid nitrogen. The ground powder was homogenized and immersed with $2.0 \mathrm{~mL}$ of

17 hexane containing guaiacol $(20 \mu \mathrm{g})$ as an internal standard. The mixture was incubated

18 at $25^{\circ} \mathrm{C}$ for $1 \mathrm{hr}$ to extract eugenol. The extracts were dried over anhydrous sodium

19 sulfate and concentrated to $100 \mu \mathrm{L}$ by passing air-flow. The concentrated extracts were

20 passed through a CENTRICUT W-MR column (MF $0.4 \mu \mathrm{m}$, Kurabo, Osaka, Japan) to

21 remove any residue. Eugenol and other phenylpropene volatiles were analyzed by a

22 Shimadzu GC-2014 or GCMS-QP2010 Plus equipped with a DB-5 column $(15 \mathrm{~m} \times$

$23 \quad 0.25 \mathrm{~mm}$ I.D. $\times 0.4 \mu \mathrm{m}$ film thickness, Agilent Techonologies) as previously described

24 [8]. Separation conditions were as follows: $80^{\circ} \mathrm{C}$ for the initial temperature using a

25 2-min hold, and then a temperature gradient from $80^{\circ} \mathrm{C}$ to $240{ }^{\circ} \mathrm{C}$ at $10^{\circ} \mathrm{C} \mathrm{min}{ }^{-1}$ was

26 applied, followed by a 5-min hold at $240^{\circ} \mathrm{C}$. Injection and detector temperatures were

27 set at $250^{\circ} \mathrm{C}$ and $280^{\circ} \mathrm{C}$, respectively. Eluted compounds were identified by comparing 
1 their retention time and mass fragmentation patterns with authentic standards.

2

$3 \quad L C / M S / M S$ analysis of eugenol glycosides in transgenic hybrid aspen

4 Each frozen leaf sample was powdered with a mortar and pestle. Powdered samples

$5 \quad(100 \mathrm{mg})$ were extracted with $300 \mu \mathrm{L}$ of extraction solvent (methanol containing 10

$6 \mu \mathrm{g} / \mathrm{mL}$ genistein as an internal standard). After samples had been homogenized twice

7 with a Mill MM 300 mixer (Qiagen, Hilden, Germany) at $27 \mathrm{~Hz}$ for $2 \mathrm{~min}$,

8 homogenates were centrifuged. The supernatant was removed, and an additional $300 \mu \mathrm{L}$

9 was added to the residue, and the extraction was repeated. The pooled extracts were

10 diluted with methanol to 10 times volume, and filtered through a $0.2 \mu \mathrm{m}$ PVDF

11 membrane (Whatman, Brentford, U.K.). The filtrate (10 $\mu \mathrm{L})$ was applied to LC-MS.

12 A Finnigan LTQ Orbitrap XL (Thermo Fisher Scientific, Waltham, MA) coupled 13 with an Agilent 1200 system (Agilent Technologies, Palo Alto, CA) was used for 14 LC-MS analysis. A TSK-gel column ODS-100V $(5 \mu \mathrm{m}, 4.6 \mathrm{~mm} \mathrm{I.D.} \times 250 \mathrm{~mm}$, 15 TOSOH, Tokyo, Japan) was used for separation of eugenol glycosides by mobile phase 16 consisting of $0.1 \%(\mathrm{v} / \mathrm{v})$ aqueous formic acid (solvent $\mathrm{A})$ and $0.1 \%(\mathrm{v} / \mathrm{v})$ formic acid in 17 acetonitrile (solvent B). The gradient program was as follows: 3 to $50 \%$ B for the first $1820 \mathrm{~min}, 50$ to $90 \%$ B from 20 to $40 \mathrm{~min}, 90 \%$ B from 40 to $45 \mathrm{~min}$, and $95 \%$ B from 45

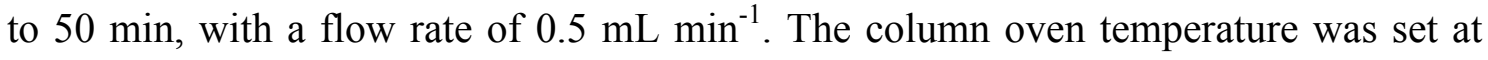

$2040^{\circ} \mathrm{C}$. The MS system was operated in the positive mode according to a previously 21 described method [17].

22 Quantification of eugenol glycosides in the leaves of hybrid aspen was performed 23 using calibration curves prepared with eugenyl primeveroside.

25 Purification of eugenyl 6-O- $\beta$-D-xylopyranosyl- $\beta$-D-glucopyranoside (eugenyl 26 primeveroside)

27 For purification of eugenyl 6-O- $\beta$-D-xylopyranosyl- $\beta$-D-glucopyranoside (eugenyl 
1 primeveroside), the fresh leaves of Camellia sasanqua were plucked at the Center for

2 Education and Research in Field Sciences, Shizuoka University (Fujieda, Shizuoka,

3 Japan) in March from 2009 to 2011. Fresh young leaves were finely chopped and

4 crushed in liquid nitrogen by a homogenizer. The fine powder was extracted three times

5 with $80 \%$ methanol and filtered. The combined extracts were concentrated in vacuo and

6 separated three times with hexane and $\mathrm{H}_{2} \mathrm{O}$. The combined aqueous fractions were

7 applied on a solid phase extract column (Oasis HLB 3 cc Vac Cartridge, Waters,

8 Milford, MA). The column was washed with $\mathrm{H}_{2} \mathrm{O}$ and eluted with $\mathrm{MeOH}$. The eluent

9 was applied to a reversed-phase HPLC column (Cosmosil 5C 18 -MS-II, 4.6 mm I.D. $\times$

$10150 \mathrm{~mm}$, Nacalai Tesque, Kyoto, Japan) with gradient elution of 15 to $70 \%$ acetonitrile

11 in $\mathrm{H}_{2} \mathrm{O}$ and detection at UV $278 \mathrm{~nm}$. The collected fractions were checked by

12 HPLC-LC/MS ( $\mathrm{m} / \mathrm{z}$ 371, negative mode), and the fractions containing eugenyl

13 primeveroside were further purified by HPLC (CAPCELL PAK $\mathrm{C}_{18}$ UG120, $4.6 \mathrm{~mm}$

14 I.D. $\times 250 \mathrm{~mm}$, Shiseido, Tokyo, Japan) to afford the target compound. The molecular

15 formula and ${ }^{1} \mathrm{H}$ and ${ }^{13} \mathrm{C}$ NMR spectroscopic data of the isolated compound was identical

16 to eugenyl primeveroside as previously described [16].

\section{Results}

19 Generation of transgenic plants

20 Previous studies showed that two enzymes, CFAT and EGS, were required to convert

21 coniferyl alcohol to eugenol [5,7]. Because detectable levels of coniferyl alcohol were 22 observed in aspen differentiating xylem [19], we rationalized that the overexpression of

23 CFAT and EGS would result in an increase in the production of eugenol in aspen.

24 Therefore, we used a mixture of two different Agrobacterium transformants [14]

25 harboring pH35GS-PhCFAT and pH35GS-PhEGS (Supplementary Fig. 1) to infect

26 hybrid aspen stem segments. We obtained more than 50 transgenic lines after repeated

27 subcultures containing hygromycin as a selective reagent. The transgene expression was 
1 checked by RT-PCR using leaves of the regenerated transformants (Fig. 2). Of these

2 lines, PhCFAT alone was expressed in 18 lines, PhEGS alone was expressed in 26 lines,

3 and both PhCFAT and PhEGS were expressed in 12 lines. All transgenic lines

4 established with the PhCFAT and/or PhEGS genes showed the same morphological

5 phenotype as wild-type plants (Supplementary Fig. 2). Some of the representative

6 transformants that survived after transplanting in pots were used for the subsequent 7 analysis.

$9 \quad$ Production of eugenol and its glycosides in transgenic aspen plants overexpressing PhCFAT and/or PhEGS

11 Leaves of transgenic lines and wild type of hybrid aspen were extracted with hexane

12 and analyzed by GC-MS for the production of eugenol and other volatile compounds.

13 GC analyses of PhEGS-overexpressing lines showed only a slight increase in eugenol

14 level in hybrid aspen as compared to that of the wild type (the eugenol content of 15 wild-type plants ranged from 6.2 to $8.8 \mu \mathrm{g} / \mathrm{g}$ fresh weight, with an average of $7.4 \pm$ $161.3 \mu \mathrm{g} / \mathrm{g}$ fresh weight, whereas for PhEGS-expressing lines, an average of $11.3 \pm$ $173.2 \mu \mathrm{g} / \mathrm{g}$ fresh weight). However, in the transgenic lines overexpressing both PhCFAT 18 and $P h E G S$ or only PhCFAT, a significant increase in the eugenol production was 19 observed, with eugenol content measured at 52.5 and $38.9 \mu \mathrm{g} / \mathrm{g}$ fresh weight on average, 20 respectively. In particular, the PhCFAT transformant no. 4 accumulated $85.1 \mu \mathrm{g}$ 21 eugenol/g fresh weight, which was 12-fold greater than that of the wild type (Fig. 3). It should be noted that no other phenylpropenes were detected in addition to eugenol in 23 the transgenic lines (data not shown).

24 Eugenol is often detected as a glycosylated form in plants [1,20,21]. Therefore, the 25 accumulation of eugenol glycosides in the leaves was analyzed using liquid 26 chromatography/mass/mass spectrometry (LC/MS/MS) (Supplementary Fig. 3). When 27 compared with the mass pattern and retention time of the standard eugenyl 
1 primeveroside, glycosylated eugenol was detected as a monoglycoside and a 2 diglycoside in all of the transformant as well as the wild-type aspen plants. In the

3 PhCFAT-overexpressing line, the maximal increase in both eugenol monoglycoside and 4 diglycoside of 22 and 3.5-fold, respectively, was observed over the levels seen in 5 wild-type plants, and the PhCFAT and PhEGS double transformants exhibited 7.5 and 6 2.5-fold increases over wild type (Fig. 4). In contrast, the level of eugenol glycosides

7 from $P h E G S$ single transformants was only slightly higher than the wild-type level. A

8 similar accumulation pattern between the level of eugenol glycosides and its aglycone 9 was observed among all the transformants (Fig. 3, 4).

10 Since transgenic aspen leaves expressing only PhCFAT showed a significant 11 increase in eugenol biosynthesis, we checked to see if aspen leaves already express an 12 endogenous gene encoding EGS. This was a reasonable hypothesis particularly in light 13 of the observations that non-transgenic aspen leaves already synthesize low levels of 14 eugenol (Fig. 3). A search of the database 15 (http://compbio.dfci.harvard.edu/cgi-bin/tgi/gimain.pl?gudb=poplar) identified several 16 aspen cDNAs that encode proteins with high identity to bona fide EGS proteins (Table 17 1). Moreover, transcripts of some of these genes were identified in leaf RNA (Table 1). 18 As expressing PhCFAT by itself led to a large increase in eugenol production in 19 hybrid aspen, we next examined whether transforming tobacco and Arabidopsis plants 20 with PhCFAT would also result in increased eugenol production. The T2 generation 21 plants of transformants with pMDC32-PhCFAT (Fig. 2) were analyzed, and, in contrast 22 to hybrid aspen, no significant increase in the eugenol production was observed in both 23 transgenic tobacco and Arabidopsis harboring PhCFAT as compared to the wild type 24 (Supplementary Table 1).

\section{Discussion}

27 Recently, Hoffman et al [11] reported that the expression of basil EGS gene or petunia 
1 IGS (isoeugenol synthase) in strawberry fruit, which is rich in anthocyanins (pigments

2 that are derived from $p$-coumaroyl $\mathrm{CoA}$ ), led to a large increase in the levels of eugenol,

3 chavicol, isoeugenol, and anol. The presence of phenylpropene glycosides was not

4 examined. Based on these results, they concluded that the strawberry fruit already

5 expresses the putative acyl transferases that produce monolignol acetates but lacks the

6 final enzyme (EGS or IGS) for phenylpropene biosynthesis. This conclusion was

7 supported by the observation that $p$-coumaryl acetate accumulated in the transgenic strawberry fruits expressing a CHS-RNAi construct (suppressing chalcone synthase).

In our investigation in aspen we have observed that expressing PhCFAT by itself

10 greatly increased the production of eugenol, and expressing PhEGS in addition to

11 PhCFAT also caused an increase in eugenol production, but at a lower level compared 12 with $P h C F A T$ alone, while expressing $P h E G S$ by itself resulted in only a minor increase 13 in eugenol levels over those seen in wild-type aspen plants. These observations suggest 14 that, as in strawberries, aspen has a latent ability to biosynthesize eugenol, but that the 15 limiting enzyme in aspen is not EGS but CFAT. Thus, the introduction of PhCFAT to 16 aspen plants led to a much higher flux for monolignol acetates and hence a significant 17 increase in eugenol biosynthesis. This conclusion is consistent with the observations 18 that (1) wild-type aspen leaves contain some eugenol, (2) their vascular tissue 19 (including the prominent veins in the leaves) is known to synthesize monolignols, and

20 (3) genes with significant sequence identity to bona fide EGS enzymes have been found 21 to be expressed in aspen leaf tissue.

22 The expression of PhCFAT did not lead to the production of other phenylpropenes 23 such as chavicol and methoxyeugenol in the transgenic aspen leaves (data not shown). 24 One possible explanation for this observation is that, even though PhCFAT was present 25 in the transgenic plants, the PhCFAT activities against $p$-coumaryl alcohol and sinapyl 26 alcohol is lower than its activity with coniferyl alcohol, as previously reported [7], or 27 that the levels of $p$-coumaryl alcohol and sinapyl alcohol were not sufficient for 
1 chavicol or methoxyeugenol formation in planta, suggesting that the phenylpropene

2 production is dependent on in vivo production of monolignols and their acetates.

3 In addition to the increase in eugenol levels, PhCFAT overexpression with/without

$4 \quad P h E G S$ expression also led to a proportional increase in the levels of eugenol glycosides

5 in transgenic hybrid aspen (Fig. 4). Because eugenol is generally toxic to plant cells as

6 well as to herbivores, free eugenol is often stored in the compartmentalized tissue such

7 as trichomes [22] or is converted to its glycosylated forms that can be stored in vacuoles.

8 It was previously reported that tomato fruits and leaves of Camellia sasanqua contain

9 glycosylated eugenol rather than its aglycone [1,20]. In this study, we used eugenyl

10 6-O-( $\beta$-D-xylopyranosyl)- $\beta$-D-glucopyranoside (eugenyl primeveroside) isolated from $C$.

11 sasanqua leaves as a standard. However, the mass spectroscopic analysis indicated that

12 the aspen eugenol diglycosides are not identical to that of C. sasanqua leaves although

13 the MS/MS spectra are very similar [Supplementary Fig. 3 (E) and (F)].

14 In contrast to the situation in aspen, where small amounts of eugenol were already 15 found in wild-type plants and the introduction of PhCFAT alone greatly increased these 16 amounts, wild-type tobacco and Arabidopsis plants did not contain any eugenol, and the 17 introduction of PhCFAT did not lead to any eugenol synthesis. This observation 18 suggests that these plants are missing multiple enzymes in the pathway to 19 phenylpropene in the leaf tissues examined.

20 This report demonstrates that the overexpression of PhCFAT in transgenic hybrid 21 aspen leads to higher levels of eugenol and eugenol glycosides. For in vivo 22 phenylpropene production, the supply of the endogenous monolignols and their acetates 23 in the host plants is clearly a prerequisite in attempt to manipulate phenylpropene levels.

24 In future work it would be of interest to investigate if the increased levels of eugenol in 25 the transgenic plants correlates with antimicrobial and acaricidal activities.

\section{Acknowledgement}


1 We thank Dr. Kazufumi Yazaki of Kyoto University for his valuable advice and Ms.

2 Naoko Tsue of Kyoto University for her technical assistance. We also acknowledge Dr.

3 Taku Demura of Nara Institute of Science and Technology, Dr. Csaba Koncz of

4 Max-Planck Institute for Plant Breeding Research, and Dr. Björn Sundberg of Umeå

5 Plant Science Centre for providing pH35GS vector, A. tumefaciens GV3101::pMP90,

6 and $P$. tremula $\times$ tremuloides T89, respectively. We also thank DASH (Development

7 and Assessment of Sustainable Humanosphere) system of Research Institute for

8 Sustainable Humanosphere, Kyoto University. This study was supported in part by the

$9 \quad$ Shorai Foundation for Science and Technology.

10

11 References

12 [1] Y.M. Tikunov, R.C. de Vos, A.M. González Paramás, R.D. Hall, A.G. Bovy, A role 13 for differential glycoconjugation in the emission of phenylpropanoid volatiles from 14 tomato fruit discovered using a metabolic data fusion approach, Plant Physiol. 152 $15 \quad$ (2010) 55-70.

17 [2] S. Birtić, C. Ginies, M. Causse, C.M. Renard, D. Page, Changes in volatiles and 18 glycosides during fruit maturation of two contrasted tomato (Solanum lycopersicum) 19 lines, J. Agric. Food Chem. 57 (2009) 591-598.

21 [3] P. Zhang, E. Zhang, M. Xiao, C. Chen, W. Xu, Enhanced chemical and biological activities of a newly biosynthesized eugenol glycoconjugate, eugenol $\alpha$-D-glucopyranoside, Appl. Microbiol. Biotechnol. 97 (2013) 1043-1050.

[4] C. Pasay, K. Mounsey, G. Stevenson, R. Davis, L. Arlian, M. Morgan, D. 26 Vyszenski-Moher, K. Andrews, J. McCarthy, Acaricidal activity of eugenol based compounds against scabies mites, PLoS One 5 (2010) e12079. 
2 [5] T. Koeduka, E. Fridman, D.R. Gang, D.G. Vassão, B.L. Jackson, C.M. Kish, I.

3 Orlova, S.M. Spassova, N.G. Lewis, J.P. Noel, T.J. Baiga, N. Dudareva, E. Pichersky,

4 Eugenol and isoeugenol, characteristic aromatic constituents of spices, are

5 biosynthesized via reduction of a coniferyl alcohol ester, Proc. Natl. Acad. Sci. USA

$6 \quad 103(2006)$ 10128-10133.

7

8 [6] G.V. Louie, T.J. Baiga, M.E. Bowman, T. Koeduka, J.H. Taylor, S.M. Spassova, E.

9 Pichersky, J.P. Noel, Structure and reaction mechanism of basil eugenol synthase,

$10 \quad$ PLoS One 2 (2007) e993.

11

[7] R. Dexter, A. Qualley, C.M. Kish, C.J. Ma, T. Koeduka, D.A. Nagegowda, N. Dudareva, E. Pichersky, D. Clark, Characterization of a petunia acetyltransferase involved in the biosynthesis of the floral volatile isoeugenol, Plant J. 49 (2007) $265-275$.

[8] T. Koeduka, G.V. Louie, I. Orlova, C.M. Kish, M. Ibdah, C.G. Wilkerson, M.E. Bowman, T.J. Baiga, J.P. Noel, N. Dudareva, E. Pichersky, The multiple phenylpropene synthases in both Clarkia breweri and Petunia hybrida represent two

20 distinct protein lineages, Plant J. 54 (2008) 362-374.

[9] T. Koeduka, T.J. Baiga, J.P. Noel, E. Pichersky, Biosynthesis of t-anethole in anise: characterization of t-anol/isoeugenol synthase and an O-methyltransferase specific for a C7-C8 propenyl side chain, Plant Physiol. 149 (2009) 384-394.

26 [10] T. Koeduka, I. Orlova, T.J. Baiga, J.P. Noel, N. Dudareva, E. Pichersky, The lack 27 of floral synthesis and emission of isoeugenol in Petunia axillaris subsp. parodii is 
1 due to a mutation in the isoeugenol synthase gene, Plant J. 60 (2009) 961-969.

2

3 [11] T. Hoffmann, R. Kurtzer, K. Skowranek, P. Kiessling, E. Fridman, E. Pichersky, W. Schwab, Metabolic engineering in strawberry fruit uncovers a dormant biosynthetic pathway, Metab. Eng. 13 (2011) 527-531.

7 [12] M. Kubo, M. Udagawa, N. Nishikubo, G. Horiguchi, M. Yamaguchi, J. Ito, T.

8 Mimura, H. Fukuda, T. Demura, Transcription switches for protoxylem and 9 metaxylem vessel formation. Genes Dev. 19 (2005) 1855-1860.

11 [13] M.D. Curtis, U. Grossniklaus, A gateway cloning vector set for high-throughput functional analysis of genes in planta, Plant Physiol. 133 (2003) 462-469.

14 [14] L. Li, Y. Zhou, X. Cheng, J. Sun, J.M. Marita, J. Ralph, V.L. Chiang, 15 Combinatorial modification of multiple lignin traits in trees through multigene cotransformation. Proc. Natl. Acad. Sci. USA 100 (2003) 4939-4944.

[15] A. Bent, Arabidopsis thaliana floral dip transformation method, Methods Mol. Biol. 343 (2006) 87-103.

21 [16] R.B. Horsch, J.E. Fry, N.L. Hoffmann, D. Eichholtz, S.G. Rogers, R.T. Fraley, A simple and general method for transferring genes into plants, Science, 227 (1985) 1229-1231.

25 [17] Y. Iijima, Y. Nakamura, Y. Ogata, K Tanaka, N. Sakurai, K. Suda, T. Suzuki, H. 26 Suzuki, K. Okazaki, M. Kitayama, S. Kanaya, K. Aoki, D. Shibata, Metabolite 27 annotations based on the integration of mass spectral information, Plant J. 54 (2008) 
$1 \quad 949-962$

2

3 [18] M. Straubinger, H. Knapp, N. Watanabe, N. Oka, H. Washio, P. Winterhalter, 4 Three novel eugenol glycosides from rose flowers, Rosa damascena Mill, Natural $5 \quad$ Product Letters 13 (1999) 5-10.

6

7 [19] S. Suzuki, N. Sakakibara, L. Li, T. Umezawa, V.L. Chiang, Profiling of 8 phenylpropanoid monomers in developing xylem tissue of transgenic aspen (Populus 9 tremuloides). J. Wood Sci. 56 (2010) 71-76.

10

11 [20] C.R. Parks, K. Kondo, T. Swain, Phytochemical evidence for the genetic 12 contamination of Camellia sasanqua Thunberg, Japan J. Breed. 31 (1981) 168-182.

14 [21] S. Hammami, M.L. Ciavatta, H.B. Jannet, G. Cimino, Z. Mighri, Three phenolic and a sterol glycosides identified for the first time in Matthiola longipetala growing

18 [22] D.R. Gang, T. Beuerle, P. Ullmann, D. Werck-Reichhart, E. Pichersky, Differential production of meta hydroxylated phenylpropanoids in sweet basil peltate glandular

20 trichomes and leaves is controlled by the activities of specific acyltransferases and 21 hydroxylases, Plant Physiol. 130 (2002) 1536-1544.

\section{Table legend}

24 Table 1. Genes encoding EGS-like proteins in Populus EST databases.

\section{Figure legend}

27 Figure 1. Formation of phenylpropenes in transgenic plants. 
1 Eugenol is formed with the subsequent reactions by coniferyl alcohol acetyltransferase

2 (CFAT) and eugenol synthase (EGS) from coniferyl alcohol. Eugenol is glycosylated by

3 the endogenous glycosyltransferase (GT). Lignins are produced by the polymerization

4 of monolignols such as $p$-coumaryl alcohol and coniferyl alcohol. The minor pathway

5 leading to hydroxyphenyl lignin was indicated by a broken arrow. Chalcone synthase

6 (CHS) catalyzes the formation of flavonoids and anthocyanins. The phenylpropene

7 pathway was indicated by a broken line.

8

9 Figure 2. Expression of coniferyl alcohol acetyltransferase and eugenol synthase in 10 independent transformants.

11 Semi-quantitative polymerase chain reaction analysis confirmed PhCFAT and PhEGS

12 transcript levels in transgenic hybrid aspen. Actin expression levels were used as an

13 internal control. Expression of PhCFAT mRNAs in transgenic tobacco (SR1) and

14 Arabidopsis (Col-0) were also shown. WT indicates wild type.

15

16 Figure 3. The accumulation of eugenol in transgenic hybrid aspen.

17 (A) Representative gas chromatograms of hexane extracts from aspen leaves.

18 (B) Level of eugenol accumulated in aspen leaves.

20 Figure 4. The accumulation of eugenol glycosides in transgenic hybrid aspen.

21 The amount of the glycosides is represented as relative abundance (Wild type $=1$ )

22 (A) Level of eugenol monoglycoside in aspen leaves.

23 (B) Level of eugenol diglycoside in aspen leaves.

\section{Supplementary data}

26 Supplementary Figure 1. The constructs for overexpressing of PhCFAT and PhEGS

27 under control of the $35 \mathrm{~S}$ promoter. 
1 (A) The constructs used for overexpression of PhCFAT and PhEGS in hybrid aspen.

2 (B) The constructs used for overexpression of PhCFAT in tobacco and Arabidopsis.

3

4 Supplementary Figure 2. Morphology of transgenic plants overexpressing PhCFAT 5 and/or PhEGS.

6

7 Supplementary Figure 3. LC/MS/MS analysis of eugenol glycosides produced by 8 transgenic hybrid aspen overexpressing PhCFAT.

9 (A) HPLC chromatogram of eugenol monoglycoside extracted from PhCFAT 10 transformants.

11 (B) HPLC chromatogram of eugenol diglycoside extracted from PhCFAT 12 transformants.

13 (C) HPLC chromatogram of authentic eugenyl primeveroside.

14 The metabolic products and authentic eugenol glycoside were analyzed by LC/MS/MS

15 using selected ion monitoring at $\mathrm{m} / z 371$ for eugenol monoglycoside (D) and at $\mathrm{m} / \mathrm{z} 503$

16 for eugenol diglycoside (E) and authentic eugenyl primeveroside (F).

17

18 Supplementary Table 1. Production of eugenol in transgenic plants with overexpression 19 of PhCFAT. 
Table 1. Genes encoding EGS-like proteins in Populus EST databases.

\begin{tabular}{clccc}
\hline Sequence ID & Annotation & Length & Identity to PhEGS & Expressed in leaf tissue \\
\hline TC142239 & Isoflavone reductase-like & $(\mathrm{bp})$ & $(\%)$ & yes \\
TC147936 & Leucoanthocyanidin reductase-like & 1991 & 76 & yes \\
TC138212 & Phenylcoumaran benzylic ether reductase-like & 1321 & 41 & yes \\
TC141798 & Isoflavone reductase-like & 1211 & 55 & no \\
TC145463 & Phenylcoumaran benzylic ether reductase-like & 1178 & 77 & no \\
TC144261 & Phenylcoumaran benzylic ether reductase-like & 1030 & 78 & yes \\
TC148965 & Pinoresinol-lariciresinol reductase-like & 976 & 49 & no \\
CK320793 & Phenylcoumaran benzylic ether reductase-like & 812 & 76 & no \\
TC154756 & Phenylcoumaran benzylic ether reductase-like & 754 & 74 & no \\
\hline
\end{tabular}

Table 1. Koeduka et al. 


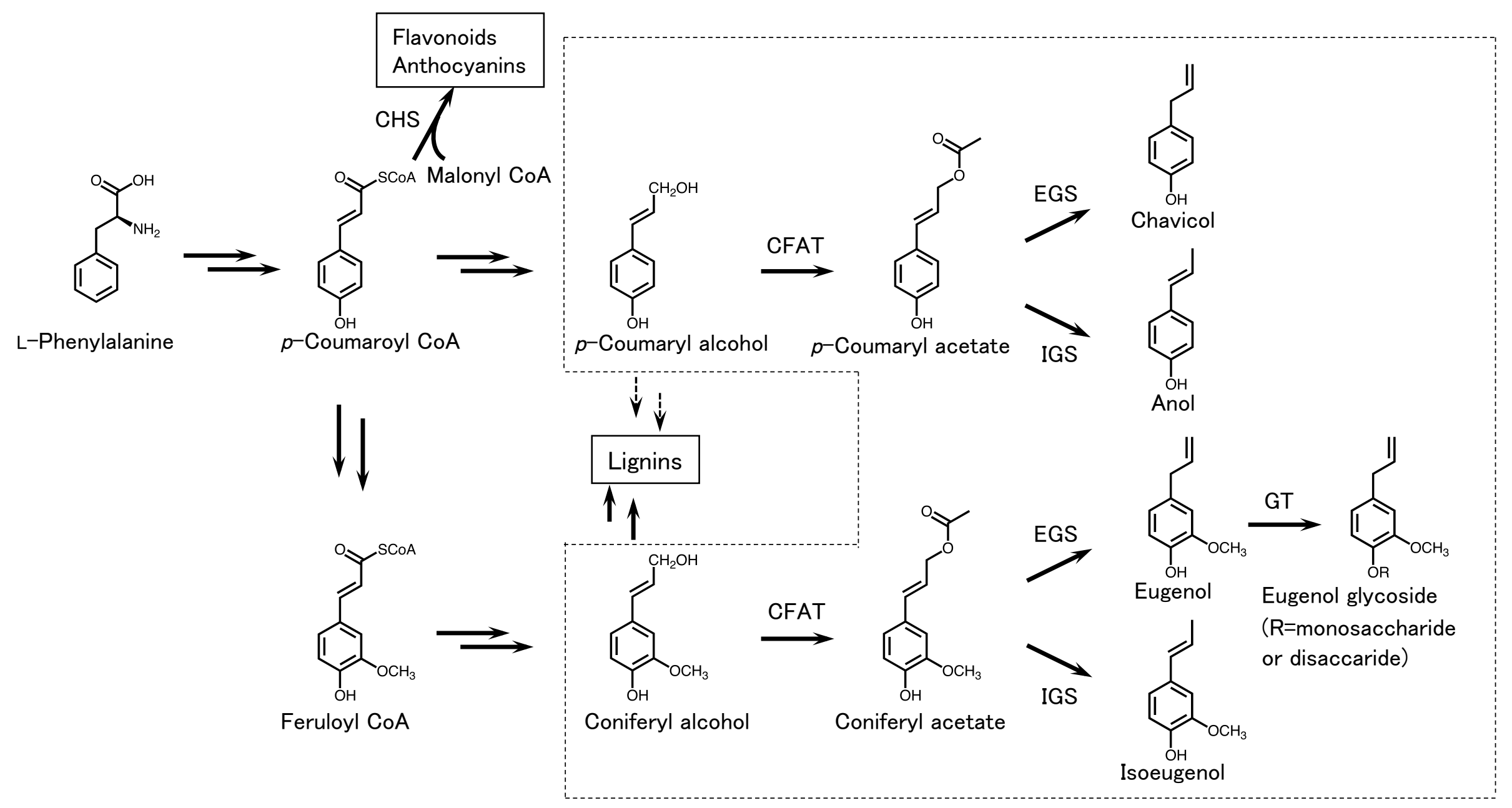

Figure 1. Koeduka et al. 

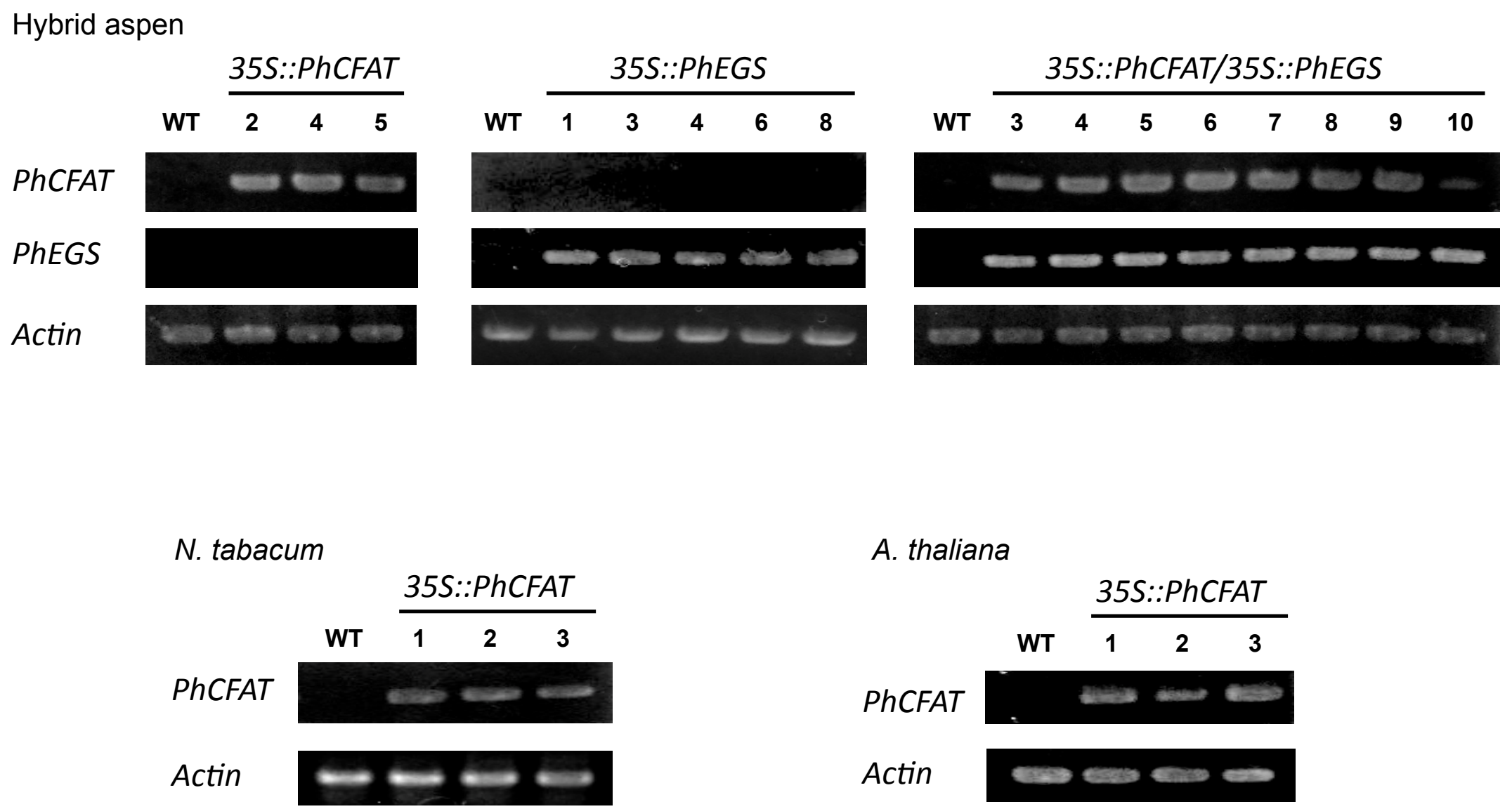
A. thaliana

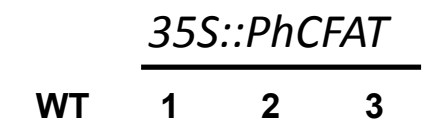
PhCFAT mas ans
Actin
$m \infty$

Figure 2. Koeduka et al. 

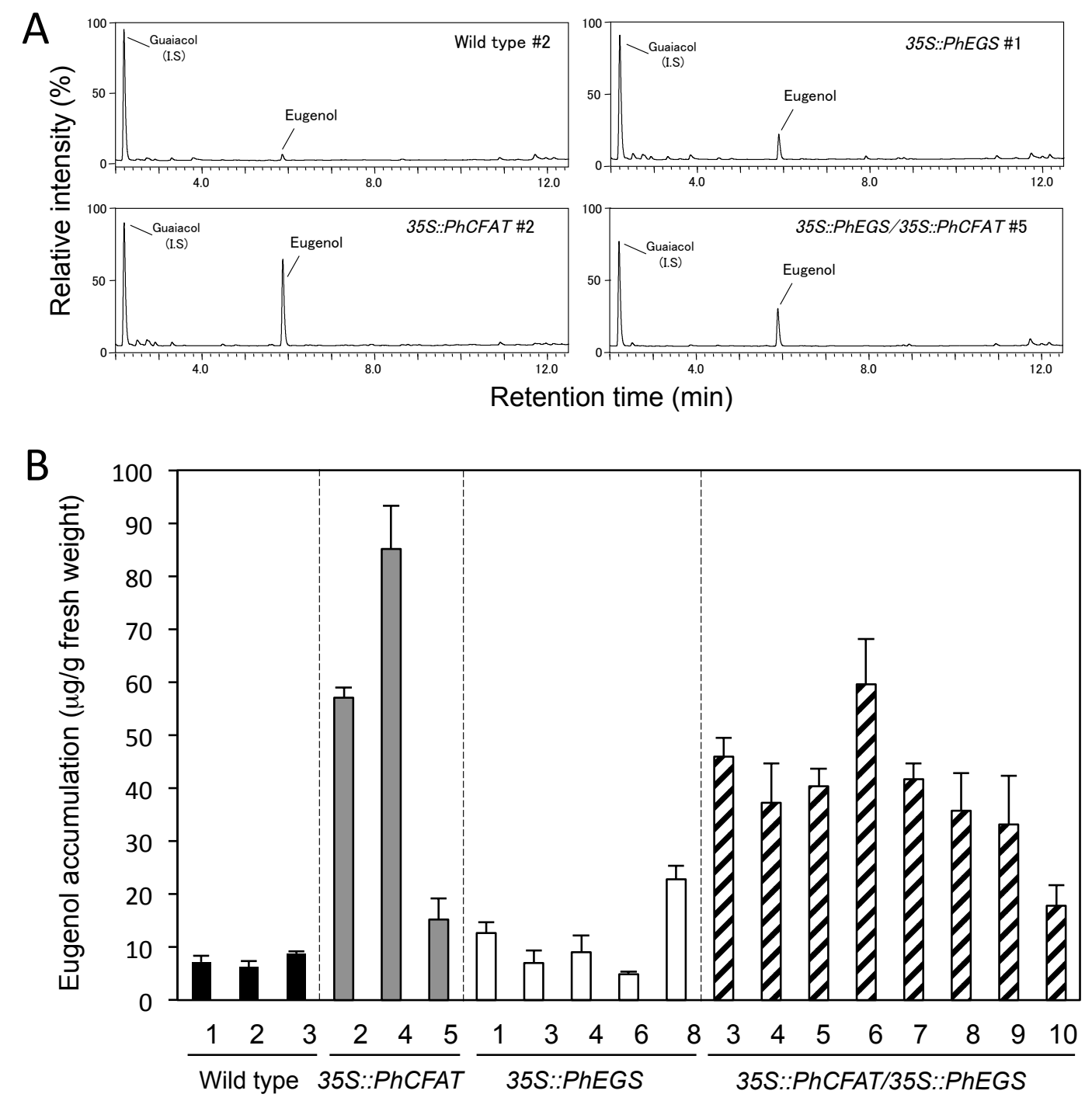

Figure 3. Koeduka et al. 

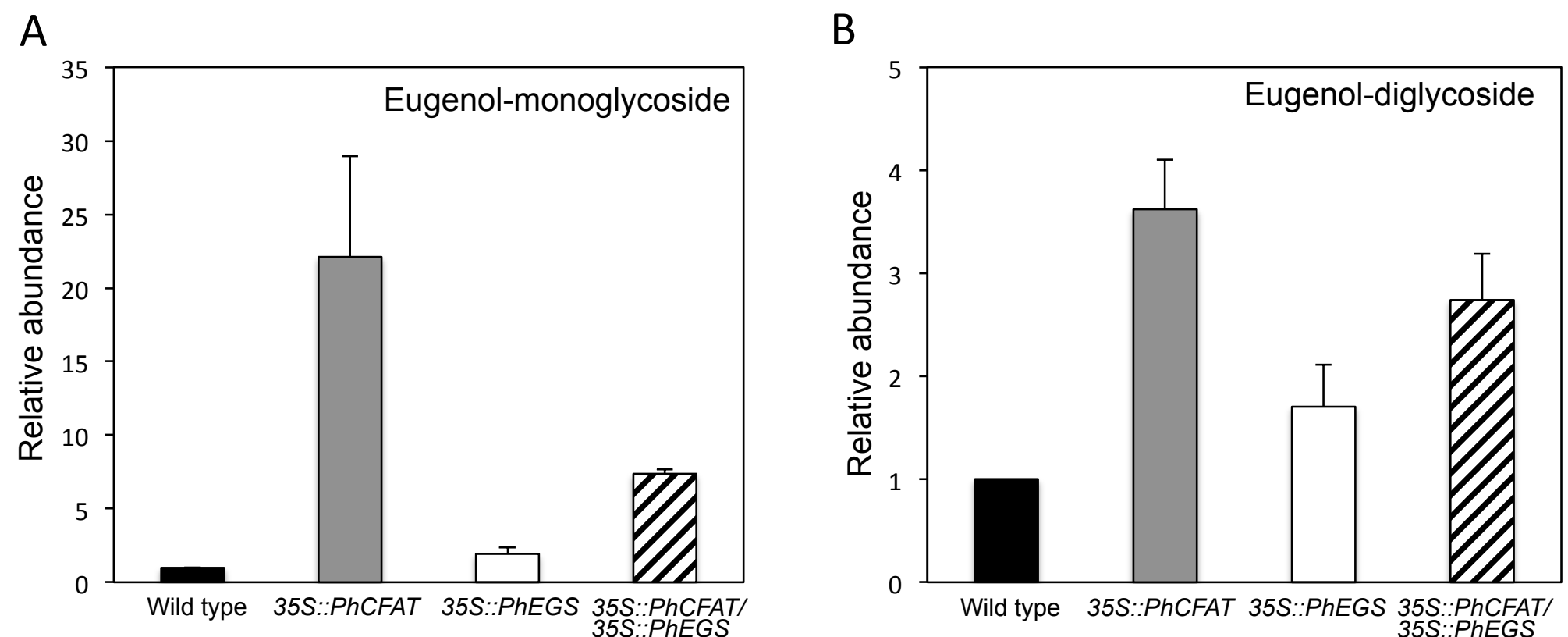

Figure 4. Koeduka et al. 


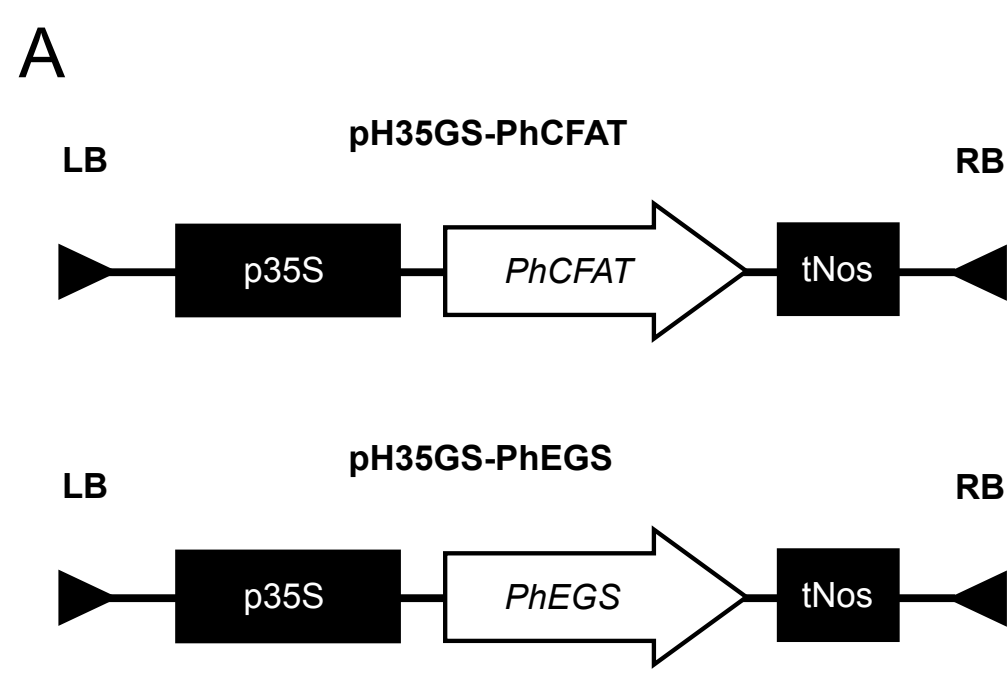

B

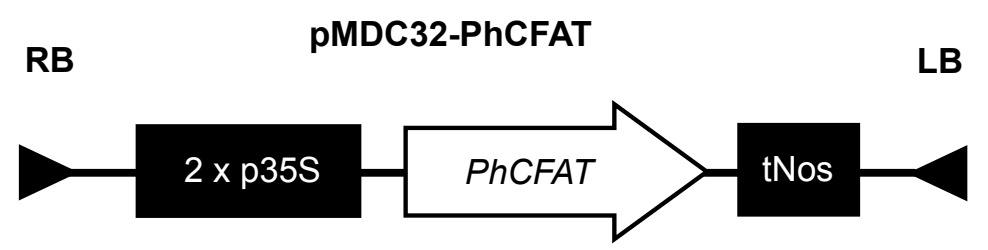

RB

Supplementary Fig. 1. Koeduka et al. 


\section{Hybrid aspen}

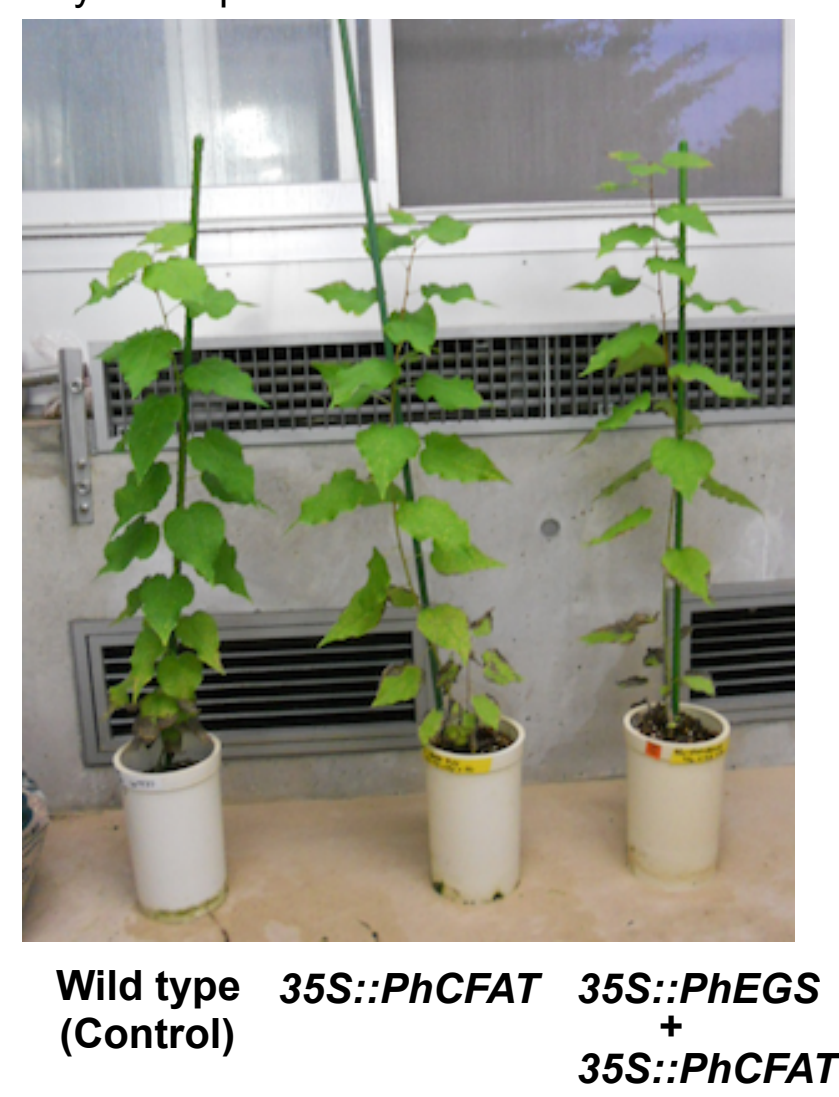

Arabidopsis

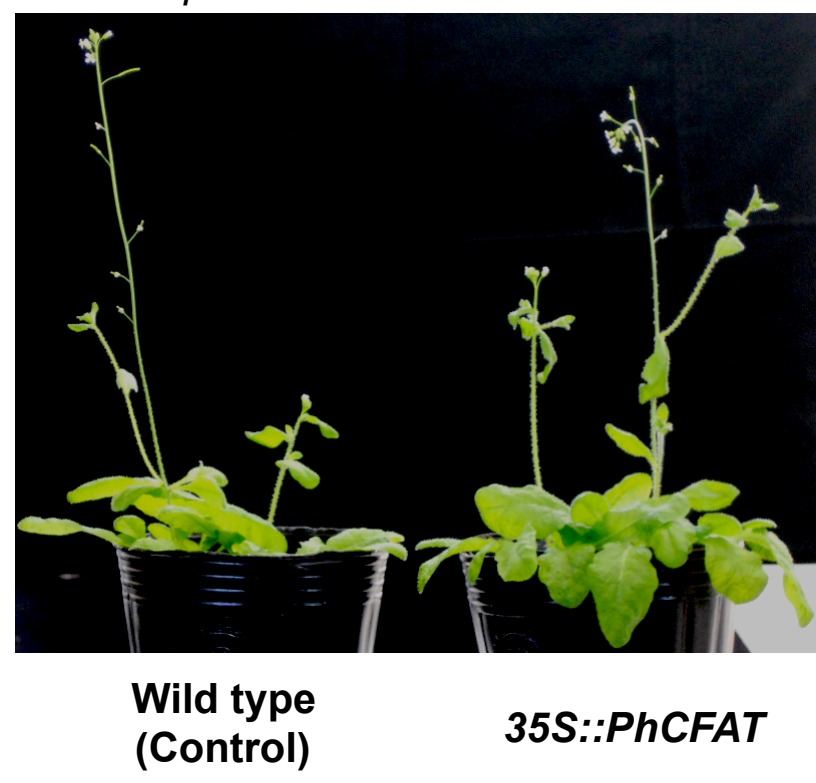

Tobacco

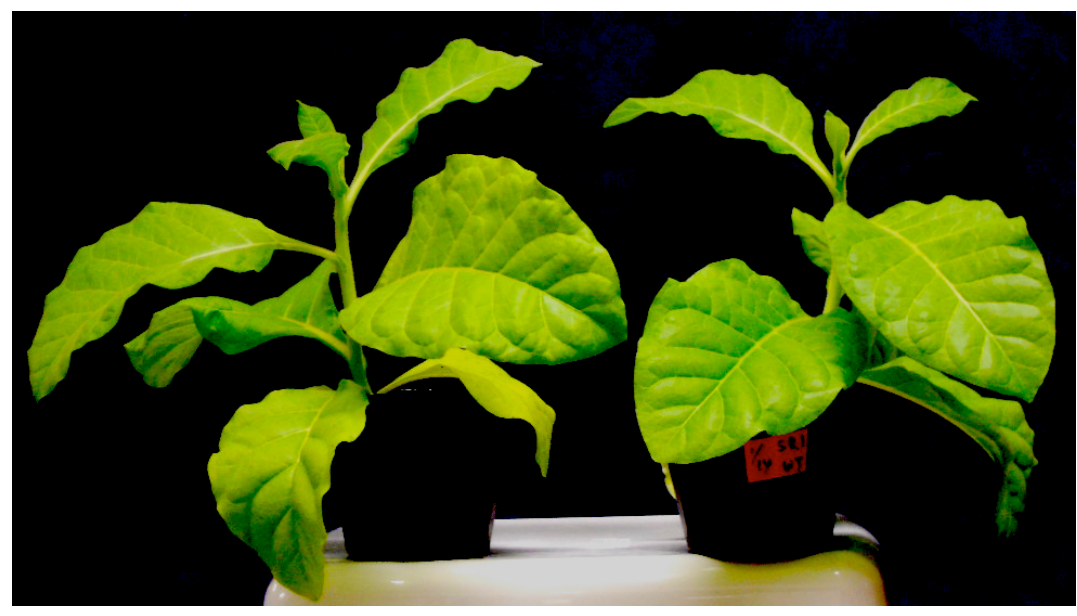

Wild type (Control)

Supplementary Fig. 2. Koeduka et al. 


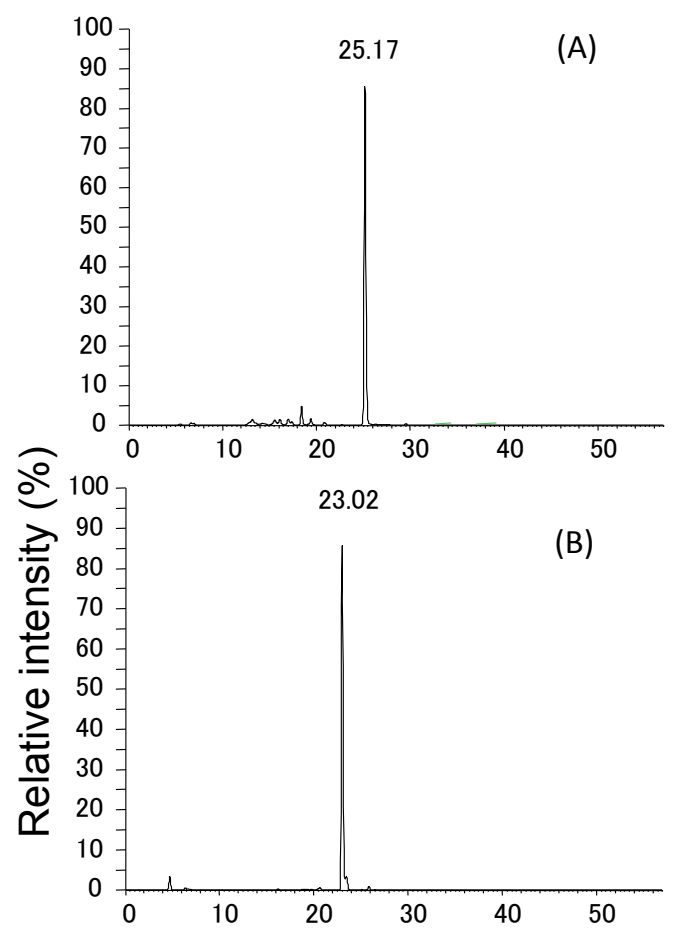

(D)

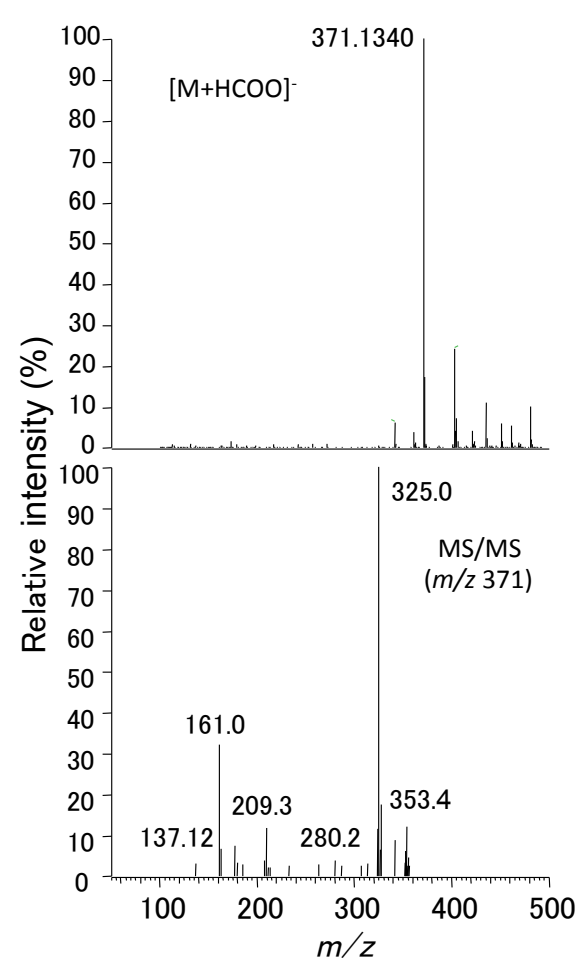

(E)

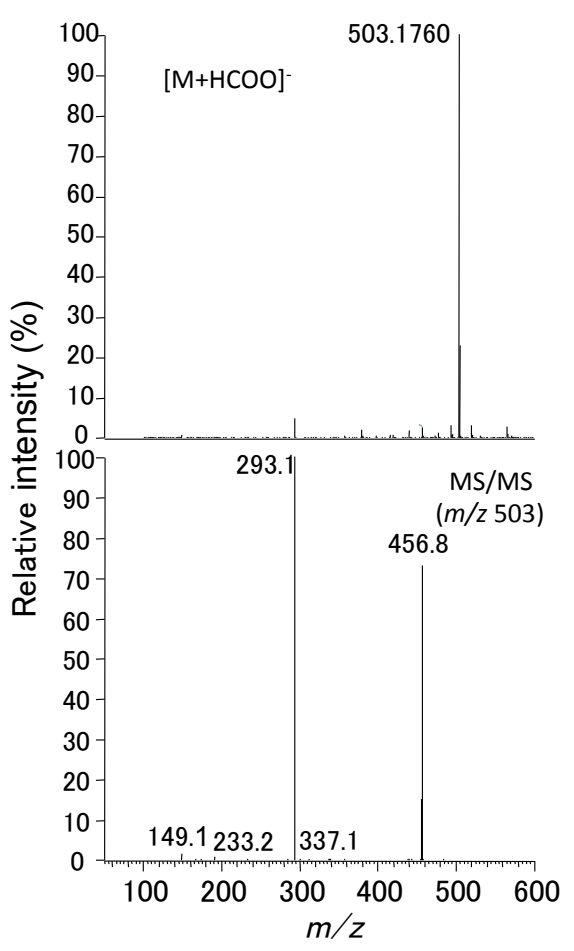

(F)

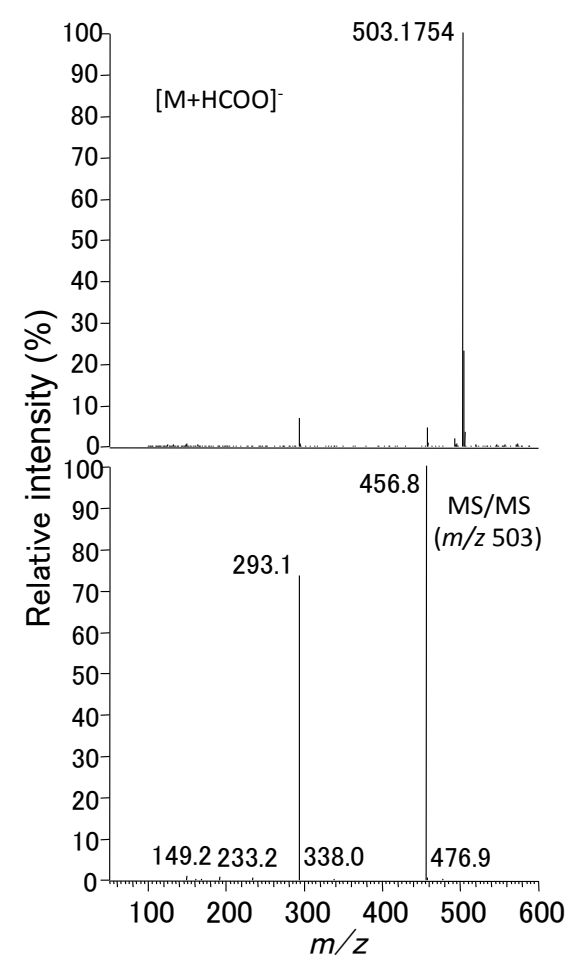


Supplementary Table 1. Production of eugenol in transgenic plants with overexpression of PhCFAT.

\begin{tabular}{lcc}
\hline Host plants & Line & Produced eugenol \\
\hline Hybrid aspen & wild type & Mg/g fresh weight \\
& PhCFAT & $52.4 \pm 20.3$ \\
Tobacco & wild type & n.d. \\
& PhCFAT & n.d. \\
Arabidopsis & wild type & n.d. \\
& PhCFAT & n.d. \\
\hline
\end{tabular}

n.d., Not detectable 